\title{
Guest Editorial: Special Issue on Computing Frontiers
}

\author{
Preface \\ Martin Schulz ${ }^{1} \cdot$ Kento Sato ${ }^{2}$ \\ Received: 22 October 2020 / Revised: 23 October 2020 / Accepted: 28 October 2020 \\ (C) The Author(s) 2021
}

\section{Introduction/Conference Information}

This special issue collects extended versions of the highscored papers of the 2019 edition of the ACM International Conference on Computing Frontiers (CF19). The CF19 conference was held April 30-May 2 in Sardinia, Italy.

CF19 is an eclectic, collaborative community of researchers who investigate emerging technologies in the broad field of computing: our common goal is to drive the scientific breakthroughs that transform society. Technology is experiencing revolutions in memory devices and systems, networks, electronic device production, machine learning, data analytics, cloud computing, techniques to improve power and energy efficiency, and systems portability/wearability, to name but a few areas. New application domains that affect everyday life are emerging, especially in the era of highly interconnected and collaborative cyber-physical systems. Boundaries between the state-of-the-art and revolutionary innovation constitute the frontiers that mark the advances of science, engineering, and information technology. Early research that envisions future technologies provides the bases that allow novel materials, devices, and systems to become mainstream. Collaborative efforts among researchers with different expertise and backgrounds enables revolutionary scientific breakthroughs that lead to innovative solutions over a wide spectrum of computer systems,

\footnotetext{
Martin Schulz

schulzm@in.tum.de

$\triangle$ Kento Sato

kento.sato@riken.jp

1 Computer Architecture and Parallel Systems, Technical University of Munich, Technische Universität, MünchenBoltzmannstraße 3, 85748 Garching, Germany

2 High Performance Big data Research Team, Center for Computational Science, RIKEN, 7-1-26 Minatojima-minami-machi, Chuo-ku, Kobe, Hyogo 650-0047, Japan
}

from embedded and hand-held/wearable devices to supercomputers and data centers.

In this wide context, we have assembled a collection of articles that cover a variety of topics: from programming frameworks for parallel architectures, to novel applications and computing platforms, to more traditional architectural themes. Among the 19 regular papers accepted at the conference (out of 61 total regular papers submissions) we have invited authors of the seven most representative, in terms of quality and topics, to submit extended versions for this special issue. From these seven papers, after a rigorous peer-review process according to the Journal of Signal Processing Systems standards, we have selected four to appear in this Special Issue.

\section{Identifying the Author Contributions}

The first article, "IterML: Iterative Machine Learning for Intelligent Parameter Pruning and Tuning in Graphics Processing Units" by Xuewen Cui and Wu-chun Feng, proposes an approach that uses statistical analysis with iterative machine learning (IterML) to prune and tune hyperparameters to achieve better performance.

The second article, "Decomposition algorithms for solving NP-hard problems on a quantum annealer" by Elijah Pelofske, Georg Hahn and Hristo Djidjev, proposes a general decomposition algorithm for NP-hard graph problems aiming to find an optimal set of vertices on a quantum annealer.

The third article, "Zero-Power Defense Done Right: Shielding IMDs from Battery-Depletion Attacks" by Muhammad Ali Siddiqi, Wouter A. Serdijn and Christos Strydis, presents essential design considerations for employing Zero-Power Defence (ZPD) techniques in commercial Implantable Medical Devices (IMDs), which offers a critical review of ZPD techniques found in literature and, subsequently, gives crucial recommendations for developing comprehensive ZPD solutions. 
The last article, "A Memory Reliability Enhancement Technique for Multi Bit Upsets" by Alexandre Chabot, Ihsen Alouani, Réda Nouacer and Smail Niar, proposes a new memory reliability techniques referred to as DPSR: Double Parity Single Redundancy. DPSR is designed to enhance computing systems resilience to Single Bit Upsets (SBU) and Multiple Bit Upsets (MBU).

\section{Summary/Conclusions}

These four papers provide a detailed compilation of the diverse range from programming frameworks for parallel architectures, to novel applications and computing platforms, to more traditional architectural themes. We hope that the community will find this special issue to be a useful and interesting collection of articles.

Guest Editors:

Martin Schulz

Kento Sato
Funding Open Access funding enabled and organized by Projekt DEAL.

Open Access This article is licensed under a Creative Commons Attribution 4.0 International License, which permits use, sharing, adaptation, distribution and reproduction in any medium or format, as long as you give appropriate credit to the original author(s) and the source, provide a link to the Creative Commons licence, and indicate if changes were made. The images or other third party material in this article are included in the article's Creative Commons licence, unless indicated otherwise in a credit line to the material. If material is not included in the article's Creative Commons licence and your intended use is not permitted by statutory regulation or exceeds the permitted use, you will need to obtain permission directly from the copyright holder. To view a copy of this licence, visit http://creativecommons.org/licenses/by/4.0/.

Publisher's Note Springer Nature remains neutral with regard to jurisdictional claims in published maps and institutional affiliations. 